Volume 8. No. 7, July 2020

International Journal of Emerging Trends in Engineering Research

Available Online at http://www.warse.org/IJETER/static/pdf/file/ijeter128872020.pdf

https://doi.org/10.30534/ijeter/2020/128872020

\title{
Service Discovery in Mobile Ad-Hoc Environments: A Solution Space Analysis
}

\author{
Murimo Bethel Mutanga \\ Department of Information and Communication Technology, Mangosuthu University of Technology, Umlazi, \\ Durban, South Africa, mutangamb@mut.ac.za
}

\begin{abstract}
The rapid and pervasive growth of Cloud computing and SOA has given rise to a lot of other remarkable offshoots. One interesting feature of this trend has been the emergence of the Ad-hoc Mobile Cloud (AMC) paradigm. Ad-hoc Mobile Cloud allows users to collaborate and share web services in a platform that enables mobile devices to play the role of service providers. This peculiar functional architecture that relies on resource-limited devices to host and perform discovery operations introduces another dimension to the service discovery challenge in conventional mobile environments. The conventional Cloud and Mobile Cloud environments leverage on the limitless cloud storage and computing power, therefore, techniques designed for such environments are generally resource-unaware. Consequently, the traditional service discovery mechanisms designed for Cloud computing cannot be adopted into AMC. In this review, we present an analysis of how service discovery solutions in Ad-hoc Mobile Cloud have adapted to meet the unique challenges of this resource-constrained environment. We classified, at a generic level, the solution space of the current service discovery techniques in Ad-hoc mobile cloud, and the identify opportunities for advancing research in the domain.
\end{abstract}

Key words: Ad-hoc mobile environments, cloud computing, context-awareness, dynamic environments, service discovery.

\section{INTRODUCTION}

The cloud computing paradigm which is inspired by Service Oriented Architecture and Service-Oriented Computing concepts has undergone interesting evolutions in recent decades. This evolutionary trend has seen new dimensions of the cloud computing paradigm such as Mobile Cloud and, in the last decade, Cloudlet [1], and Ad-hoc Mobile Cloud computing [2] emanate from the traditional cloud model. The emergence of these Cloud variants has been motivated by several factors which include, but not limited to resource considerations, cost, convenience, and application environment [3].

For instance, the integration of mobile devices into the conventional Cloud architecture to form Mobile Cloud
Computing (MCC) was essentially intended to expand e-services' market landscape to cover the ever-growing mobile consumer-base. More so, aside from adding the flexibility of mobility to the traditional cloud computing, MCC is also targeted at addressing challenges such as availability, resource limitation, security, and scalability, which are characteristic of mobile computing environments [4], [5].

However, being a service provisioning environment, the requirements for service discovery and invocation in addition to cost efficiency in MCC still proves to be a significant challenge to mobile clients. Fundamentally, these challenges stem from the inherent nature of mobile devices and the mobile environment. For instance, in MCC, mobile devices require an intermediary infrastructure like access point or satellite as well as a stable and strong Internet connection to be able to sufficiently discover and access any Cloud resource. Such requirement has varied implication on the client-side: wireless connection from the mobile device to the Cloud leads to high energy consumption, weak or absence of Internet connection cause inaccessibility of the Cloud, and the hop distance between mobile devices and Cloudlet or Cloud being accessed can introduce high latency [6], [7].

Furthermore, concerning service discovery and consumption, service relevance is influenced by the dynamic context. On the other hand, discovery efficiency hinges on the capabilities of the device consuming the service [8], [9]. Despite the popularity of earlier Cloud models, these challenges are yet to be fully addressed.

Addressing these aforementioned challenges has motivated the advent of various Ad-hoc Mobile Cloud computing paradigms. This paradigm is a complementary approach to MCC, which consists, instead, in enabling either direct service provisioning between mobile devices or indirect service provisioning a community mobile devices in collaboration with a Cloudlet [10], [11]. These devices exploit self-organizing networks to support direct communication with each other [12]. Therefore AMC is a network of mobile devices that act as Cloud providers by enabling each device to expose its resources to others within the network [13]. That is, in the AMC scenario, a mobile node can host web services that are discovered and invoked by other mobile nodes within the ad-hoc network [14]-[16].

In realizing these emerging mobile computing paradigms, significant research is ongoing toward optimizing service discovery techniques to suit the particular characteristics of these environments. The previous review works in this field 
have mostly focused on the wider cloud or mobile cloud computing while only discussing service discovery mechanisms as a component of their discourse [17], [4], [15]. Moreover, these reviews provide a more general understanding of services discovery approaches but service discovery techniques in the emergent ad-hoc mobile environments (AMEs) in specific are either not explored in-depth or they are left out.

Therefore, in this review, our main goal was to describe the solution approaches adopted in the current literature to address the service discovery in a manner that addresses the inherent challenges in AMEs. We focused on papers that formulated or implemented techniques involving the use of context-awareness, cloud-assisted mechanisms, and other lightweight approaches to achieve service in AMEs. We approached the review following the Grounded Theory Literature Review (GTLR) method [18], which enabled us to explore scholarly work and identify future research directions. First, we developed a framework for analyzing existing works is ADMs as illustrated in Table 1. The framework served as a template that highlights the landscape of the key designed requirements for services discovery in AMEs, namely, resource-intensiveness, context-awareness, request adaptation, and relevancy factor. These core requirements foreground the critical considerations for ensuring the operational performance of service discovery processes and the efficiency of service discovery in AMEs. Second, we used these design requirements to categorize the techniques that appear in the review studies and to analyze the overall implications of such techniques on AMEs. Based on insight from the analysis, we identified future research opportunities to advance research in service discovery mechanisms for AMEs.

Therefore, we aim to complement the existing body of knowledge by showing a picture of the research work done in service discovery that targeted at addressing the unique challenges of AMEs. Such an overview should be useful for researchers because the evaluation of the existing techniques in line with the core design requirements of AMEs provides useful insights into the current challenges.

In the rest of the paper, we offer the methodological details of the review in Section 2, and the framework for review analysis is presented in section 3. A summary of the reviewed papers is given in section 3 . Then in section 4 , we used the criteria provided in our framework as the basis to describe the various techniques advanced in the reviewed papers and their implication to AMEs. The paper is concluded in section 5 with a discussion in the context of how the current solution space of service discovery techniques in AMEs presents unexplored future research opportunities.

\section{THEORETICAL GROUNDING}

This review adopted the Grounded Theory Literature Review method as described in Wolfswinkel's work [18]. This review methodology is designed to enhance rigor in the overall process of conducting a literature review study. The GTLR enables researchers to transform existing content from carefully selected papers to empirical data that is then coded and systematically analyzed to create new insights or extend existing knowledge. There are five stages in the GTLR method: i) scope definition - outlining of inclusion and exclusion criteria, delineation of information sources, and creating search terms; ii) retrieval of potential papers; iii) review sample formation -filter and then select papers into the sample papers to be reviewed based on the abstract of the papers included in the preliminary sample; iv) in-depth paper analysis; v) presentation of the emerging trends from the papers based on defined categories. These stages are outlined in the rest of this section.

\subsection{Scope of the Reviews}

We delineate the scope of this review as the literature that discussed the design and implementation of service discovery techniques that are targeted at AMEs. This delineation was intended to enable us to concentrate on how the unique design requirements for service discovery in AMEs were addressing in the current solution space. While many papers were potentially relevant in the general context of service discovery, we considered them to be out of the scope of this study if the paper: i) did not implement service discovery techniques in any of the following subsets of our scope, namely, AMC, AMC blended with Cloudlet, or IoT blended with other AMC architectures. For example, an ontology-supported context-aware service model for cloud computing is presented in [19]; ii) did not explicitly address at least one of the core design requirements for efficient service discovery in AMEs as highlighted our framework. In one of such papers, Palmieri discussed a random walk-based scalable search technique for pervasive computing environments [20]; and iii) if they only presented an architectural description or framework such as in the works reported in [21] and [22].

\subsection{Retrieval of Papers - Sources of Information}

A comprehensive literature searches was conducted with the research team by first developing a list of relevant phrases constructed from keywords. These phrases as shown in Table 1 were framed with the help of researchers who are well vest with the terminologies of the field and the phrases were then used to search two major academic bibliography search engines, name, Google Scholar, and Microsoft Academic. These indexing databases are predominantly meant for searching scholarly content. Moreover, Google Scholar particularly is like a superset of Scopus and Web of Science, the most extensive academic databases, as it can return about $89 \%$ and $93 \%$ of Scopus and Web of Science citations respectively [23]. Due to time and the large volume of identified papers that were often returned on each query, we 
devised a means to constrain out search queries to return only relevant papers. To achieve this, we enclosed keywords in double-quotes and also used the symbolic Boolean operator "+" to join the quoted keywords to form phrases. Also, considering the rapid technological evolution in the ubiquitous computing domain, we reviewed paper published between 2010 to 2019. Our thinking is that the earlier techniques may not be very relevant considering the change in technology.

As depicted in Table 1, a total of 159,190 papers matched our different search phrases after querying the selected academic search engines. On each search operation, the researchers decided on the suitability of the returned papers based on whether their topic aligns with the delineated focus area for this study and formed a preliminary review sample. By this approach, we intended to trim the number of retrieved papers. For example, when searching with the phrase "context-aware service discovery", 23300 papers were returned but only 98 of them were included in the preliminary sample. This result pre-selected papers were transformed into a spreadsheet further selection analysis.

Table 1: Search Phrases with Corresponding Returned and Selected Papers

\begin{tabular}{l|c|c|c}
\multicolumn{1}{c|}{ Search phrase } & Total retrievals & Relevant by topic & Relevant by abstract \\
\hline Service discovery pervasive environments & 20700 & 66 & 5 \\
Service discovery in ad-hoc mobile cloud & 17800 & 85 & 4 \\
Context-aware service discovery & 23300 & 98 & 8 \\
Resource-aware service discovery & 2990 & 56 & 2 \\
Adaptive service discovery & 52000 & 48 & 3 \\
Context-aware service selection in pervasive & 16300 & 61 & 7 \\
environments & 14600 & 77 & 2 \\
Energy-aware service discovery techniques & 63500 & 34 & 1 \\
Proactive service discovery & 159190 & 525 & 20
\end{tabular}

\subsection{Final Paper Selection}

Through several iterations among some authors who are grounded with research in pervasive environments, selected 525 papers were further scrutinized by reading the abstracts. This enabled the researches to ensure that only papers that explicitly met the requirements outlined in our framework for analysis were drafted into the final review sample. In some cases where a paper's abstract did not provide all the key information, the researcher speed-read the paper for further details to ensure potential papers were not rejected prematurely. This second round of scrutiny resulted in an overall review sample of 20 papers out of the initial 252 preliminary selections.

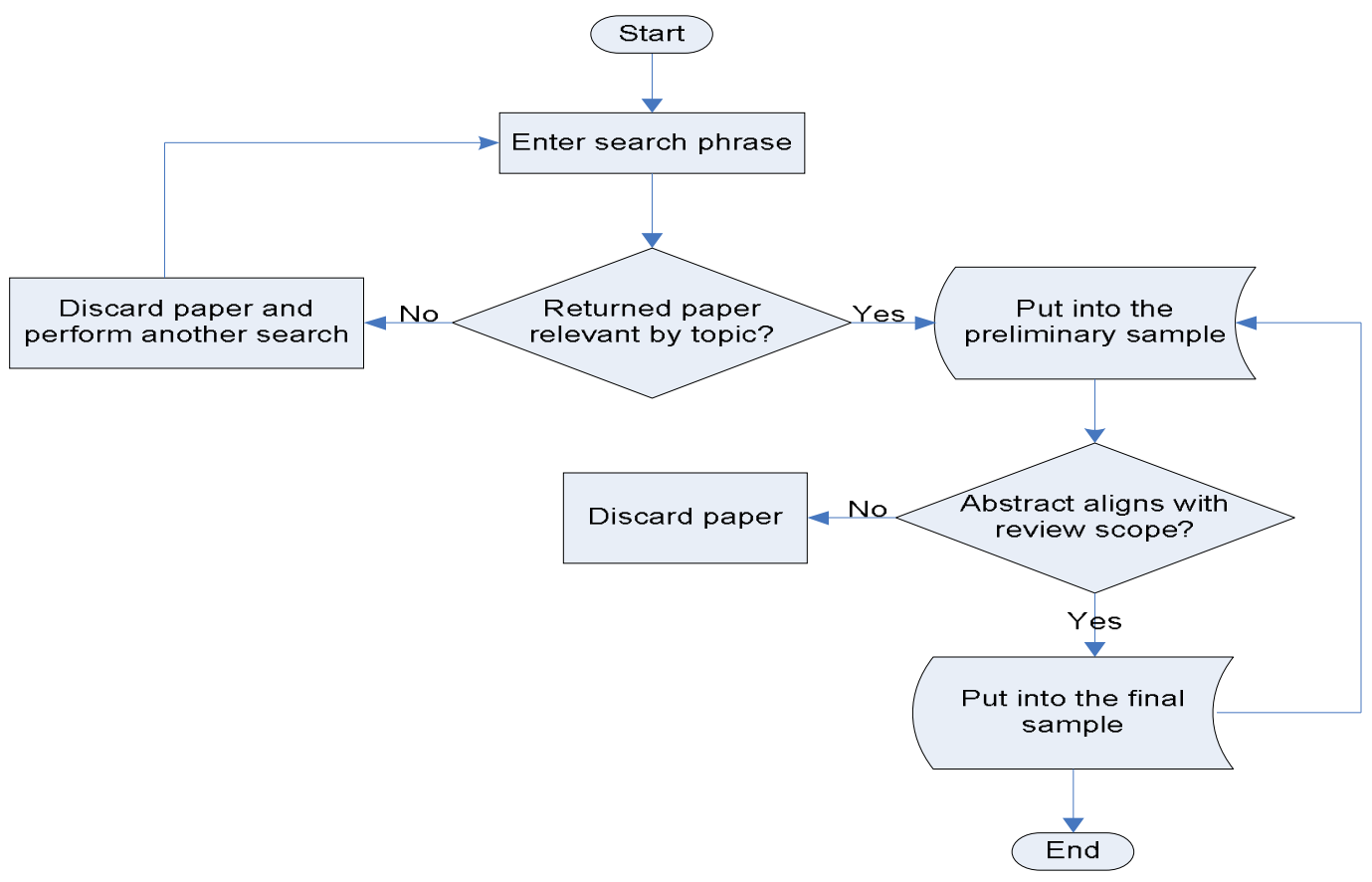

Figure 1: The Search and Selection Process

Essentially, we looked at key paragraphs on the abstracts that highlighted the problem being solved, the techniques 
adopted, and the rationale of employing such techniques. Therefore, abstracts that failed to provide clarity on these key issues were excluded from the final list of papers. Based on this final screening approach, 12 papers were excluded from the selected 32 as indicated in Table 1 and a flowchart representation of the search and selection process is given in Figure 1.

\section{FRAMEWORK FOR REVIEW ANALYSIS}

Any piece of software that is exposed over the internet by utilizing the standardized XML messaging framework is known as a web service [24]. This standard for e-service provisioning has grown in popularity because of its ability to support interoperability between machine-to-machine interaction over a network.

Table 2: Framework for Analysis

\begin{tabular}{|l|l|}
\hline Resource-intensiveness & $\begin{array}{l}\text { Service discovery mechanisms for AMEs should be lightweight i.e., adopt techniques that do } \\
\text { not weigh down the constrained resources of mobile devices at the matchmaking level. }\end{array}$ \\
\hline Context-awareness & $\begin{array}{l}\text { AMEs service discovery mechanisms should have capabilities to respond to contextual } \\
\text { changes in the environment to discover relevant services. }\end{array}$ \\
\hline Request adaptation & $\begin{array}{l}\text { Due to storage and other limitations like screen view, AMEs should employ adaptive service } \\
\text { request and retrieval options (service request is first adapted based on certain parameters so } \\
\text { that only services that meet such conditions are retrieved. }\end{array}$ \\
\hline Relevancy factor & $\begin{array}{l}\text { To give more value to the concept of service personalization, services discovery in AMEs } \\
\text { should incorporate techniques autonomously prioritizes retrieved services using clients' } \\
\text { context information (person or device). }\end{array}$ \\
\hline
\end{tabular}

\section{REVIEW ANALYSIS}

During the analysis stage, the papers in the review sample were read again and a coding scheme was developed iteratively that enabled the organization of the themes for discussion. The iteration in developing the scheme was because as more papers were read, new and related insights
The concept of web services grew from the Remote Procedure Call mechanism (RPC) in the distributed computing environment to become the core driver of cloud services [25]. And due to the key advantage offered by this concept, coupled with the fascinating development of smart devices and mobile technologies, its scope of application expanding beyond the traditional cloud [26]. However, providing such services in dynamic and resource-constrained environments requires the design of techniques that addresses the unique requirements of such environments [27]. After a preliminary study of the current literature, we formulated a framework that highlights these core requirements that enhances service discovery in AMEs [15]. This framework was used as the yardstick for our analysis of the findings of this review. were gained that sometimes led to the alteration of earlier codes or the addition of new ones. Therefore, to ensure that the coding scheme covered the scope of analysis for this review as highlighted in the framework of Table 2, papers were read multiple times. Then based on the framework, the different studies were categorized and comparatively analyzed, reflecting on the overall implications of the adopted

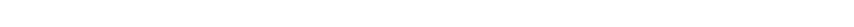
technique and the potential gaps.

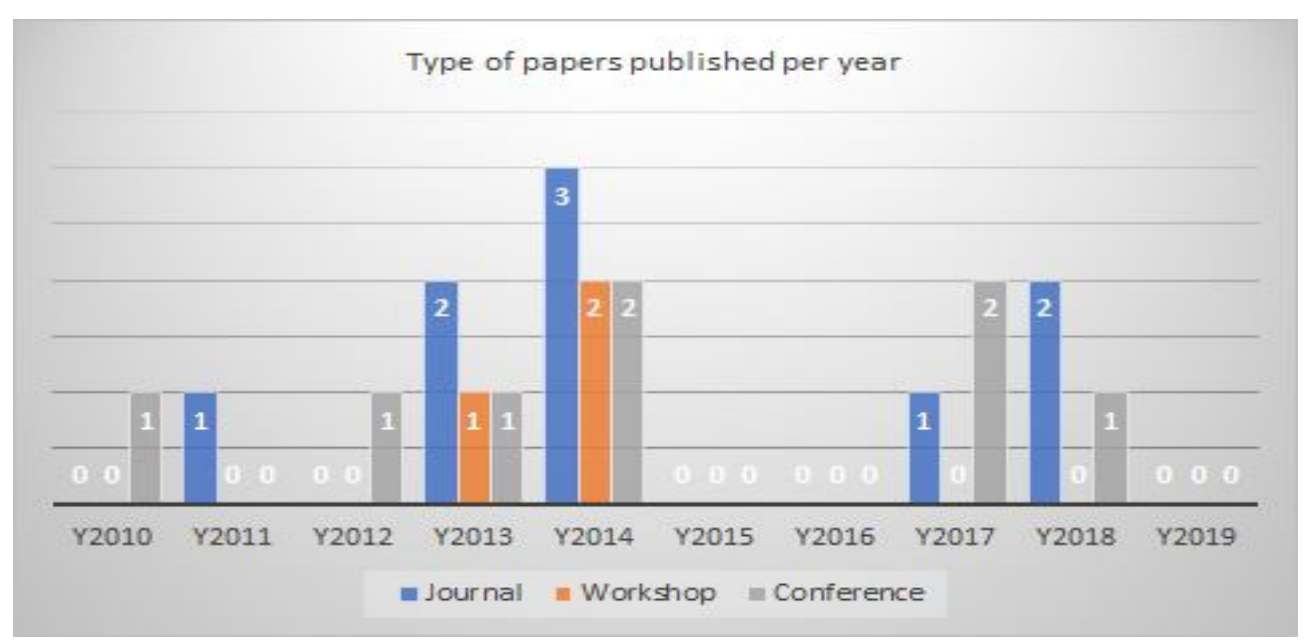

Figure 2: Review Papers Characterization 


\subsection{Characterization of the Reviewed Papers}

As shown earlier in Table 1, there were 20 papers included in the final review sample. And when Characterized by type, it is depicted in Figure that this review sample was comprised of three worship/symposium papers, eight conference papers, and nine journal articles. In terms of the volume of publication per year, 2014 was represented with the highest

\subsection{The Design Focus of Discovery Techniques}

From Table 2 we demonstrate that the techniques developed in the current literature to address the challenge of service discovery in AMEs have targeted all the design requirements that were highlighted in our framework for analysis. By this, we provide the insight that there is a huge emphasis on the use of the various dimension of context in the current solution space of service discovery in AMEs. Furthermore, we provide a clearer understanding of the level of research attention that each of these requirements has received in the recent decade. For instance, the user-centric dimension of context-awareness number of publications in the final sample of papers, with seven publications, out of which three we journals. The second-highest number of papers was recorded in 2013, having four publications in all, while three papers each were published in 2017 and 2018. The other months either had one paper each or none.

is the most predominantly employed approach to service personalization, followed by the device-centric dimension. On the other hand, request or service adaptation is the least considered design requirement.

From these findings, we observed that while the idea of context-awareness appears to be the common denominator in the design of efficient service discovery techniques in AMEs, it has also been variously modeled and applied in the current literature. However, the overall goal remains the same facilitating the discovery of relevant service by driving the concept of service personalization [28].

Table 3: Design Focus of Service Discovery Techniques

\begin{tabular}{|c|c|c|c|c|c|c|c|c|c|c|c|}
\hline \multirow{2}{*}{\multicolumn{2}{|c|}{ Design requirement }} & \multicolumn{10}{|c|}{ The design focus of techniques } \\
\hline & & 2010 & 2011 & 2012 & 2013 & 2014 & 2015 & 2016 & 2017 & 2018 & 2019 \\
\hline \multirow{3}{*}{$\begin{array}{l}\text { Context-awarenes } \\
\mathrm{s}\end{array}$} & Device-centred & - & - & - & 2 & 4 & - & - & 1 & 3 & - \\
\hline & User-centred & 1 & 1 & 1 & 4 & 6 & - & - & 2 & - & - \\
\hline & $\begin{array}{l}\text { Situation-centre } \\
d\end{array}$ & 1 & - & - & 2 & 5 & - & - & - & 1 & - \\
\hline \multicolumn{2}{|c|}{ Request/service adaptation } & - & - & - & 1 & 2 & - & - & & 3 & - \\
\hline \multicolumn{2}{|l|}{ Relevancy factor } & - & 1 & 1 & 1 & 4 & - & - & 1 & - & - \\
\hline
\end{tabular}

also promoted the ad-hoc mobile cloud paradigm where

\subsection{Device-centred Context}

Due to the dynamic nature of AMEs, the relevance of discovered services is highly context-dependent [2]. This dependence coupled with the diversity of the characteristics of the constituents of AMEs and their requirements, the need to expand the conventional scope of what constitutes context to include device characteristics have been highly advocated [11], [29].

This advocacy seems to be gaining significant research attention as half of the reviewed papers proposed techniques that employed the device context approach toward service personalization. These techniques involved the incorporation of different device parameters as context information [2], [30]-[38]. And based on the approach taken, we further grouped this context dimension into three subcategories of design techniques, namely, lightweight semantics, cloud mediated, and resource-awareness:

\section{A. The Lightweight Approach}

Following the fascinating advancement in wireless technologies and the capabilities of mobile devices, web services have not only become global business driver but it has mobile devices are empowered to serve as both web services providers and requesters [39]. To support effective mobile web service discovery, four of the reviewed papers [34], [35], [38], [40] employed the lightweight approach, which involved the use of techniques that allowed the design of optimized or less resource-intensive, semantic-based tools such as IoT-Lite and WSMO-Lite that facilitated the capturing of device capabilities as context parameters. The central idea here was to ensure that service discovery

operations are handled by mobile devices, independent of the cloud.

Generally, the lightweight approach was reported to have enhanced the discovery of relevant mobile web services by recording high precision and recall rates. Although the techniques are believed to be optimized for resource-constrained environments, the papers reviewed did not report on the resource implication on mobile devices.

\section{B. The Cloud Mediated Approach}

In the cloud mediated technique, the emphasis on the need to ease off the resource burden that is associated with service discovery operations by utilizing the cloud as a ubiquitous interface to bridges the computationally intensive 
requirements between mobile requesters and providers. As reported in [31], [33], studies that adopted such an approach used the technique of offloading computationally intensive tasks to the cloud, based on device context information. This approach seeks to enhance the efficiency and effectiveness of service discovery in AMEs by using conventional semantic and ontology-based service discovery and matchmaking algorithms that are usually too resource-intensive for mobile devices.

Findings from the reviewed papers demonstrated that leveraging on the limitless cloud computing resources to support mobile web services discovery has a myriad of advantages which include the capacity to improve the overall service discovery response time, guarantee scalability, reduce the overhead cost of gathering and processing context information, enhance of the quality of context-awareness, among others [31].

\section{The Resource-aware Approach}

When it comes to AMEs, some authors have argued that resources of mobile devices are not only limited but also dynamic - battery power can deplete, memory and storage can get used up [2]. Therefore, discovering relevant services in such dynamic environments require discovery mechanisms that monitor and capture resource context, and subsequently used such context information to determine relevant services. Notable of works among the reviewed papers included studies reported in [2], [30], [36].

Essentially, this design focus enabled the authors to tailor services to meet the current resource capabilities of mobile devices. Apart from recording high precision and recall rates, and helping to optimize resource usage, the device-aware technique also uniquely extended the concept of service relevance redefining it as "a function of meeting both client requirements and device resource capabilities" [30], [36].

\subsection{User-centred Context}

This review shows that the current service discovery solutions are dominated by techniques that designed based on the user-centred context dimension. For example, fifteen of the twenty reviewed papers design techniques that focused on the use of diverse user-centred context information. Although most of the reviewed papers used a technique that combines user context and other context information, sic of the reviewed papers focused primarily on users' context as reported in [41]-[46]. This dominance can be explained by the fact that the earliest conceptualization of context-awareness in ubiquitous computing was driven by the need to deliver services that meet users' requirements. Another interesting trend is that the last decade has seen rapid evolution and expansion in the scope of the definition of what constitutes user context.

Aside from the fundamental user context like user preferences, user location, quality of service (QoS), network characteristics, and user environment, new subsets of the user context dimension have evolved such as user privacy and identity context [43], quality of web service (QoWS)[35].

\subsection{Situation-centred Context}

This context dimension seemed to have broadened the scope of the users' context beyond the user's geographical and technical environments to include context about users' socio-cultural environments. Examples of this context dimension were presented in the reviewed papers as security context, user activity context [41], and user social context [42]. Arming service discovery mechanisms with this kind of context information was reported to outperform mechanisms based on other context information in terms of precision on recommending web services to users. From a broader perspective, it can be concluded that this finding is suggestive of the fact that more intelligent reasoners will be required in the design of future service discovery mechanisms.

\subsection{Request and Service Adaptation}

The need to improve user experience has made the concept of adaptation popular in ubiquitous environments. The concept, as implemented in the reviewed papers is a proactive way of using context information to respond to changes in dynamic environments. Nevertheless, service/request adaptation still seems sparely adopted as a design technique in the current service discovery solution space in AMEs. As shown in Table, the use of adaptation was reported in fewer papers. Based on the approach taken to implement this technique in the reviewed papers, we classified adaptation into two:

\section{A. Proactive Adaptation}

This approach was reported in [2], [30], and [41]. Basically, the authors that adopted this approach designed mechanisms that have capabilities to monitor and extract real-time context information at the time of service request and then incorporate the context into service requests. The central goal of this approach is to optimize resource usage and improve users' experience. For example, retrieving only relevant services to services requesters stand to benefits mobile devices with limited display [2].

\section{B. Reactive Adaptation}

Traditionally, services are queried into a buffer before selection filter parameters are applied which means the discovery process is a reaction to the identification of services that might meet a user's requirement. Therefore, this technique is called reaction adaptation in this review. Because reactive adaptation is the technique commonly adopted in service filtering, fewer of the reviewed papers explicitly mentioned it as a design focus [38], [37], [32], [46]. 


\subsection{Service Relevancy Factor}

To make service selection and invocation more user-friendly, existing service discovery solutions have incorporated the design capability to automate the ranking of discovered services according to users' context. In the reviewed papers, diverse context parameters were used to compute a weighted relevancy score that is then used to rank the services in the order of suitability to the user. For example, user preferences and device capabilities were used to rank service in [2], [31], [33], [35], [41], [43], while a QoS-based ranking was adopted in [40], [47].

\section{REVIEWED SERVICE TECHNIQUES: IMPLICATION FOR AMES AND FUTURE RESEARCH}

Service discovery techniques in the review presented an interesting insight into how the different design focuses align with the core requirements for efficient service discovery in AMEs. Apart from helping to reveal the state-of-the-art in the field, this insight very importantly availed us the rich context to discuss future research opportunities. These implications and the corresponding opportunities of each technique discussed as follows:

Cloud mediated technique: Resource constraint is a major challenge facing service discovery in mobile environments. This challenge makes exploring a context-aware driven solution of pushing resource-hungry tasks to the Cloud a crucial effort. While this option can salvage the limited mobile resources and strengthen service discovery efficiency, its reliance on the cloud makes it only a complementary strategy for the AMC scenario where there is either limited or no access to the Cloud [48].

Lightweight techniques: Conventional semantic-based service discovery techniques are highly efficient but too computationally complex and resource-intensive to mobile devices [49]. Optimizing such techniques has the potential to engender efficiency and accuracy in relevant service discovery in MAEs. However, considering the special AME scenarios where resource-constrained devices serve as service providers and requesters, it becomes highly imperative to answer these questions: how much resource-intensiveness is traded off in the process of optimizing semantic web service discovery tools? How scalable will such techniques be in such special AMEs?

Resource-aware Technique: Resource-awareness remains the most crucial technique in maintaining a balance between effective and efficient service discovery in AMEs. This balance means even in such special scenarios where mobile web services are provided independently of the central cloud, the functioning of the service provisioning platform is not compromised while attempting to enhance discovery efficiency. While it offers promising results for realizing relevant service discovery in AMEs, resource-aware also offers a lead to broadening the scope of the conceptualization of device context.
User/Situation Centered Technique: There is a growing need to deliver personalized services because the mobile technology evolution is increasing introducing new complexities into the scope of context. The implication is that using single context information is no longer a viable way of achieving service personalization. Furthermore, with the emergent of IoTs, where a smart device either be a service consumer or itself a service, then the concept of "user" needs to be re-modeled in the scope of context. This will require new context models as advocated in [11].

Adaptation/Ranking Technique: As the volume of web services continue to grow and devices become smarter and more capable, the demand to render services in a smarter and more user adapted manner will grow proportionately. This imminent expectation calls for more research toward designing more robust request/service adaptation techniques that can combine multiple context information in tailoring and ranking services.

\section{CONCLUSION}

This review aimed at understanding the state-of-the-art in the service discovery solution space in AMEs with a major focus on how the current techniques employed aligned with the design requirements for effective and efficient service discovery in mobile environments. We have reviewed studies drawn from journals and conferences that reported on the implementation and evaluation of service discovery mechanisms particularly designed to address the unique challenges associated with dynamic and resource-constrained environments. The review was driven by the Grounded Theory Literature Review (GTLR) method, which enabled us to gain useful insights into the existing body of work. These insights were critical in helping us to i) analyze the current techniques in the context of the core requirements that speak to the unique characteristics of AMEs; ii) ponder on the overall implications of such techniques on AMEs; iii) articulate questions that helped point to possible research gaps that offer opportunities to advance the existing body of knowledge.

Therefore, while other studies have often presented rich content on the broader scope of cloud or mobile cloud computing, this review work contributes to the existing literature by unpacking the knowledge specifically on service discovery techniques in AMEs. Also, we contribute toward shaping the research direction in this field. In particular, we emphasized the need: i) to expand the scope of the conceptualization of device context and other context dimensions; ii) to formulate new and more comprehensive context models; iii) to design more robust architecture and discovery mechanisms that can support effective and efficient service provisioning in AMEs without depending on the cloud. 
Murimo Bethel Mutanga, International Journal of Emerging Trends in Engineering Research, 8(7), July 2020, 3675 - 3683

\section{REFERENCES}

[1] M. Satyanarayanan, Z. Chen, K. Ha, W. Hu, W. Richter, and P. Pillai, "Cloudlets: at the Leading Edge of Mobile-Cloud Convergence," in Proceedings of the 6th International Conference on Mobile Computing, Applications and Services, 2014.

[2] D. A. Egbe, B. M. Murimo, and M. O. Adigun, "A Device Resource-aware Service Discovery Solution for Ad-hoc Mobile Cloud," Int. J. u- e-Serv. Sci. Technol., vol. 10, no. 1, pp. 299-316, 2017.

https://doi.org/10.14257/ijunesst.2017.10.1.26

[3] C. Stergiou and K. Psannis, "Recent Advances Delivered in Mobile Cloud Computing's Security and Management Challenges," in Modern principles, practices, and algorithms for cloud security, Gupta, Bri., IGI Global, 2020, pp. 1-23.

[4] H. Dinh, C. Lee, and D. Niyato, "A survey of mobile cloud computing: Architecture, Applications, and Approaches," Wirel. Commun. Mob. Comput., vol. 13, no. 18, pp. 1587-1611, Dec. 2013. https://doi.org/10.1002/wcm.1203

[5] N. Fernando, S. Loke, and W. Rahayu, "Mobile cloud computing: A survey," Futur. Gener. Comput. Syst., vol. 29, no. 1, pp. 84-106, 2013.

[6] E. E. Marinelli, "Hyrax : Cloud Computing on Mobile Devices using MapReduce," M.Sc. thesis, Sch. Comput. Sci. Carnegie Mellon Univ. Pittsburgh, vol. 0389, no. September, 2009.

[7] N. Palmer, R. Kemp, T. Kielmann, and H. Bal, "Proceedings of the 10th workshop on Mobile Computing Systems and Applications," in Proceedings of the 10th workshop on Mobile Computing Systems and Applications, 2009.

[8] N. E. Rathinam, L. Parthiban, and G. Mariakalavathy, "Automatic Discovery of Relevant Web Services with Semantic Ranking," J. Appl. Sci. Eng. Technol., vol. 8, no. 22, pp. 2240-2247, 2014.

https://doi.org/10.19026/rjaset.8.1224

[9] K. Elgazzar, P. Martin, and H. S. Hassanein, "Personalized Mobile Web Service Discovery," in 2013 IEEE Ninth World Congress on Services, 2013, pp. 170-174.

[10] T. Verbelen, P. Simoens, F. De Turck, and B. Dhoedt, "Cloudlets: Bringing the cloud to the mobile user," in MCS'12 - Proceedings of the 3rd ACM Workshop on Mobile Cloud Computing and Services, 2012, pp. 29-35. https://doi.org/10.1145/2307849.2307858

[11] D. A. Egbe and B. M. Murimo, "A Resource-aware Service Discovery Architecture for Ad-hoc Mobile Cloud," Int. J. Emerg. Trends Eng. Res., vol. 8, no. 6, pp. 2402-2408, 2020.

[12] C. Y. ; K. H. ; H. Chae, "Energy Efficient Mobile Cloud Computing Powered by Wireless Energy Transfer IEEE Journals \& Magazine," Sci. World J., vol. 34, no. 5, pp. 1757-1771, 2016.
[13] K. Dejan, Y. Cao, and R. Klamma, "Mobile cloud computing: A comparison of application models," arXiv Prepr. arXiv1 107.4940, 2011. https://doi.org/10.30534/ijeter/2020/32862020

[14] D. A. Egbe, P. Mudali, M. O. Adigun, A. Akingbesote, and M. B. Mutanga, "Ad-hoc Mobile Cloud Service Discovery Based on Device Resources," South. Africa Telecommun. Networks Appl. Conf. 2015, pp. 273-278, 2015.

[15] K. Elgazzar, H. Hassanein, and P. Martin, "Mobile Web Services: State of the Art and Challenges," Int. J. Adv. Comput. Sci. Appl., vol. 5, no. 3, pp. 173-188, 2014.

[16] D. A. Egbe, A. O. Akingbesote, M. O. Adigun, and M. B. Mutanga, "Context based service discovery algorithm for ad hoc mobile cloud," in 2016 International Conference on Industrial Informatics and Computer Systems (CIICS), 2016, pp. 1-6.

[17] M. Rahimi, J. Ren, and C. Liu, "Mobile cloud computing: A survey, state of art and future directions," Mob. Networks Appl., vol. 19, pp. 133-143, 2014.

[18] J. F. Wolfswinkel, E. Furtmueller, and C. P. M Wilderom, "Using Grounded Theory as a Method for Rigorously Reviewing Literature," orsociety.tandfonline.com, vol. 22, no. 1, pp. 45-55, 2011.

[19] A. Alfazi, Q. Z. Sheng, Y. Qin, and T. H. Noor, "Ontology-Based Automatic Cloud Service Categorization for Enhancing Cloud Service Discovery," in Proceedings - IEEE International Enterprise Distributed Object Computing Workshop, EDOCW, 2015, vol. 2015-November, pp. 151-158.

[20] Francesco, "Scalable service discovery in ubiquitous and pervasive computing architectures: A percolation-driven approach," Futur. Gener. Comput. Syst., vol. 29, no. 3, pp. 693-703, Mar. 2013. https://doi.org/10.1016/j.future.2012.08.004

[21] C. Bettini, O. Brdiczka, and K. Henricksen, "A Survey of Context Modelling and Reasoning Techniques," Pervasive Mob. Comput., vol. 6, no. 2, pp. 161-180, 2010.

[22] M. Pitkanen et al., "SCAMPI: Service platform for social aware mobile and pervasive computing," in Computer Communication Review, 2012, vol. 42, no. 4, pp. 503-508.

[23] ELSE, "Google Scholar, Web of Science, and Scopus: Which is best for me?," 2019. [Online]. Available: https://blogs.lse.ac.uk/impactofsocialsciences/2019/12/ 03/google-scholar-web-of-science-and-scopus-which-is -best-for-me/. [Accessed: 27-Jun-2020].

[24] Y. Kun, Y. Kun, W. Xiao-ling, and Z. Ao-ying, "Underlying Techniques for Web Services: A Survey," J. Softw., vol. 15, no. 3, pp. 428-442, 2004.

[25] M. Kalin, Java Web Services: Up and Running, 2nd Editio. O'Reilly Media, Inc, 2013.

[26] K. Wagh and R. Thool, "A Comparative Study of SOAP Vs REST Web Services Provisioning Techniques for Mobile Host," Journal of Information Engineering and 
Applications, vol. 2, no. 5. pp. 12-16, 2012.

[27] D. A. Egbe, M. B. Mutanga, and M. O. Adigun, "Service Discovery in Ad-hoc Mobile Cloud: Contemporary Approches and Future Direction," J. Theor. Appl. Inf. Technol., vol. 90, no. 1, pp. 101-117, 2016.

[28] M. M. Tseng, R. J. Jiao, and C. Wang, "Design for mass personalization," CIRP Ann. - Manuf. Technol., vol. 59, no. 1, pp. 175-178, Jan. 2010.

https://doi.org/10.1016/j.cirp.2010.03.097

[29] E. Badidi, Y. Atif, Q. Z. Sheng, and M. Maheswaran, "On personalized cloud service provisioning for mobile users using adaptive and context-aware service composition," Computing, vol. 101, no. 4, pp. 291-318, Apr. 2019. https://doi.org/10.1007/s00607-018-0631-8

[30] D. A. Egbe and B. M. Murimo, "Resource-awareness: A Strategy for Resource Optimization and Relevant Service Discovery in Ad-hoc Mobile Cloud," J. Theor. Appl. Inf. Technol., vol. 96, no. 13, pp. 4029-4043, 2018.

[31] K. Elgazzar, H. S. Hassanein, and P. Martin, "DaaS: Cloud-based mobile Web service discovery," Pervasive Mob. Comput., vol. 13, pp. 67-84, Aug. 2014.

[32] R. Pozza, M. Nati, S. Georgoulas, A. Gluhak, K. Moessner, and S. Krco, "CARD: Context-Aware resource discovery for mobile internet of things scenarios," in Proceeding of IEEE International Symposium on a World of Wireless, Mobile and Multimedia Networks (WoWMoM), 2014.

[33] N. A. Saadon and R. Mohamad, "Cloud-based Mobile Web Service Discovery framework with semantic matchmaking approach," in 2014 8th. Malaysian Software Engineering Conference (MySEC), 2014, pp. 113-118.

[34] F. Khodadadi and R. O. Sinnott, "A Semantic-aware Framework for Service Definition and Discovery in the Internet of Things Using CoAP," in Procedia Computer Science, 2017, vol. 113, pp. 146-153. https://doi.org/10.1016/j.procs.2017.08.334

[35] N. A. Saadon and R. Mohamad, "WSMO-lite based web service discovery algorithm for mobile environment," Int. J. Adv. Soft Comput. its Appl., vol. 5, no. 3, pp. 75-90, 2013.

[36] D. A. Egbe and B. M. Mutanga, "Towards a Two-tier Context-based Service Discovery Framework for Mobile Web Services 1," Int. J. Pure Appl. Math., vol. 118, no. 18, pp. 3305-3316, 2018.

[37] B. F. M'hamed and B. Abdelkader, "Context-Aware Web Service Discovery Based on A Quantitative Similarity Measure," Int. J. Mod. Educ. Comput. Sci., vol. 8, no. 5, pp. 27-42, 2013.

[38] S. Ben Fredj, M. Boussard, D. Kofman, and L. Noirie, "Efficient semantic-based IoT service discovery mechanism for dynamic environments," in IEEE International Symposium on Personal, Indoor and Mobile Radio Communications, PIMRC, 2014, pp. 2088-2092.
[39] S. Srirama and C. Paniagua, "Mobile web service provisioning and discovery in android days," Conf. Mob. Serv., 2013.

[40] N. H. Win, J.-M. Bao, and G. Cui, "Semantic QoS Ontology and Semantic Service Ranking Approach for IoT services--《Journal of Harbin Institute of Technology 》2014年06期,” J. Harbin Inst. Technol., vol. 6, no. 3, 2014.

[41] Y. Zhao, S. Wang, Y. Zou, J. Ng, and T. Ng, "Automatically Learning User Preferences for Personalized Service Composition," in Proceedings 2017 IEEE 24th International Conference on Web Services, ICWS 2017, 2017, pp. 776-783. https://doi.org/10.1109/ICWS.2017.93

[42] M. Girolami, P. Barsocchi, S. Chessa, and F. Furfari, "A social-based service discovery protocol for mobile $\mathrm{Ad}$ Hoc networks," in 12th Annual Mediterranean Ad Hoc Networking Workshop, MED-HOC-NET 2013, 2013, pp. 103-110.

[43] M. Anisetti, C. A. Ardagna, E. Damiani, and J. Maggesi, "Security certification-aware service discovery and selection," in Proceedings - 2012 5th IEEE International Conference on Service-Oriented Computing and Applications, SOCA 2012, 2012.

[44] L. Yu, S. Luo, and A. Glenstrup, "Rough sets based context-aware service discovery framework," in Proceedings - 2010 International Conference on Service Science, ICSS 2010, 2010, pp. 167-172.

[45] M. F. Khalfi and S. M. Benslimane, "Proactive Approach for Service Discovery Using Web Service for Devices on Pervasive Computing," in 3rd International Conference on Context-Aware Systems and Applications, 2014, pp. 123-12.

[46] T. A. Butt, I. Phillips, L. Guan, and G. Oikonomou, "Adaptive and context-aware service discovery for the internet of things," in Lecture Notes in Computer Science (including subseries Lecture Notes in Artificial Intelligence and Lecture Notes in Bioinformatics), 2013, vol. 8121 LNCS, pp. 36-47.

[47] H. Artail, H. Safa, P. Salameh, S. Chedrawi, and P. El Khoury, "Quality-of-service-aware cluster-based service discovery approach for mobile ad hoc networks," Int. J. Commun. Syst., vol. 27, no. 11, p. n/a-n/a, Mar. 2013. https://doi.org/10.1002/dac.2529

[48] D. A. Egbe, "Context-Aware Discovery of Relevant Web Services in Ad-hoc Mobile Cloud," University of Zululand, South Africa, 2017.

[49] L. A. Steller, "Light-weight and Adaptive reasoning for mobile web services, Ph.D thesis, Monash University, Australia." 2010. 